\title{
Geometric Constructions with Discretized Random Variables
}

\author{
Hans-Peter Schröcker (hans-peter.schroecker@uibl.ac.at) \\ Institut für Technische Mathematik, Geometrie und Bauinformatik, Universität \\ Innsbruck, Technikerstraße 13, A-6020 Innsbruck, Austria \\ Johannes Wallner (wallner@geometrie.tuwien.ac.at) \\ Institut für Diskrete Mathematik und Geometrie, Technische Universität Wien, Wiedner \\ Hauptstraße 8-10/104, A-1040 Wien, Austria
}

\begin{abstract}
We generalize the DEnv (Distribution envelope determination) method for bounding the result of arithmetic operations on random variables with unknown dependence to higher-dimensional settings. In order to minimize both the influence of the coordinate frame and information loss we suggest a nested thicket representation for random variables and a corresponding intersection algorithm.
\end{abstract}

Keywords: discrete multivariate distributions, thickets

MSC 2000: 60E05, 65G30

\section{Introduction}

Algorithms computing with discretized random variables have been presented in a number of publications (Williamson and Downs, 1990; Berleant and Goodman-Strauss, 1998; Berleant et al., 2003; Berleant and Zhang, 2004b; Berleant and Zhang, 2004a; Regan et al., 2004). However, the algebra of imprecise numbers in its various senses often is a poor model for geometry with imprecisely defined objects. A vector of imprecise numbers, for instance, usually defines an interval-like geometric object (a bounding box, a set of bounding boxes with probability measures assigned etc.), which is not invariant with respect to geometric transformations. Furthermore accurate representation of a geometric shape may require a large number of intervals.

Using the idea of tolerance zone (Requicha, 1983) and replacing intervals by spheres, ellipsoids or general convex sets when dealing with geometric constructions (Pottmann et al., 2000; Wallner et al., 2000) provides more flexibility and at the same times allows a relatively simple geometric treatment. The computational complexity of convex sets in general is, of course, higher than that of intervals. In the 2D case it is still reasonably low.

In this article we extend the DEnv method to higher-dimensional settings with convex sets as fundamental objects. DEnv stands for "distribution envelope determination" and is a method for bounding the results of arithmetic operations on discretized random variables. It has been introduced in (Berleant and Goodman-Strauss, 1998), an implementation is described in (Berleant et al., 2003); its relation to other methods for dealing with probabilistic errors in algebraic computations is the topic of (Regan et al., 2004). It is shown that these methods are equivalent to a great extent.

(C) 2005 Kluwer Academic Publishers. Printed in the Netherlands. 
In univariate DEnv, a family of random variables is represented either by a set of intervals with associated probability masses (a thicket) or by a pair of upper and lower bounds on the random variables' cumulative distribution function (CDF). Both representations can be converted into each other. The result of an arithmetic operation on random variables is bounded by pointwise optimal distribution bounds that are found by linear programming techniques. In the original version, no assumption on the mutual dependence of the random variables is made, of course at the cost of wider distribution bounds. But it is possible to incorporate knowledge of dependence, for example independence or correlation (Berleant and Zhang, 2004a; Berleant and Zhang, 2004b).

A generalization of DEnv to multivariate settings is more or less straightforward. We do this in Section 3, after a short introduction to the univariate DEnv algorithm in Section 2. The resulting multivariate DEnv method, however, is inherently information losing because multivariate thickets and their distribution bounds are no longer equivalent. In addition, the algorithm's outcome depends on the chosen coordinate system or, equivalently, on the position and orientation of the input data: Denote the output of multivariate DEnv applied to a certain set of input data by $T$ and consider the output $T^{\prime}$ of multivariate DEnv applied to a rotated copy of the same input data. In general, $T^{\prime}$ is not a rotated copy of $T$, which is undesirable in geometric applications.

In order to minimize the loss of information and to weaken the influence of the coordinate system, we introduce the concept of nested thickets and an intersection algorithm for them in Section 4. Not all problems related to multivariate thickets can be solved by these methods, but we argue that they are good enough to make thickets a valuable tool in geometric constructions. The comprehensive example of Section 5 illustrates our ideas.

\section{Univariate thickets}

This section is meant as a short introduction to univariate thickets. For more details see (Berleant and Goodman-Strauss, 1998; Regan et al., 2004; Berleant and Zhang, 2004a).

DEFINITION 1. A univariate thicket is a finite set $T=\left\{\left(C_{i}, p_{i}\right)\right\}$ whose elements are pairs of nonempty open intervals $C_{i}=\left(a_{i}, b_{i}\right) \subset \mathbb{R}$ and associated probability values $p_{i}$ such that $\sum p_{i}=1$. Upper and lower distribution bounds of the thicket $T$ are the cumulative distribution functions

$$
U(t):=\sum_{a_{i} \leq t} p_{i}, \quad L(t):=\sum_{b_{i} \leq t} p_{i} .
$$

A thicket can be thought of as a representation of a family $\mathcal{X}$ of random variables. We say that a random variable $x$, defined over the Borel sigma algebra $\mathcal{B}$ of $\mathbb{R}$, is represented by the thicket $T$ if the cumulative distribution 


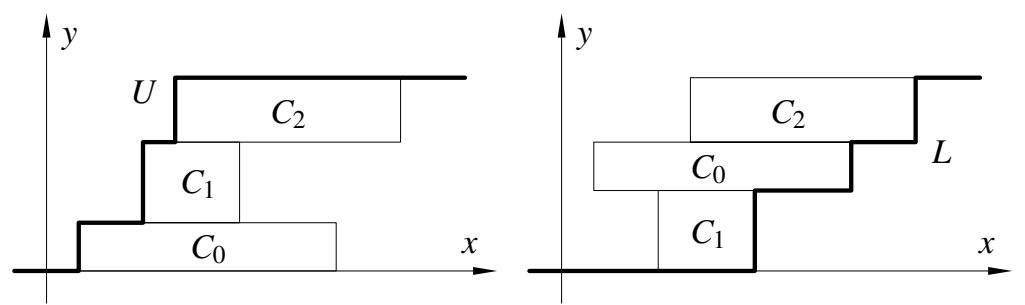

Figure 1. Construction of distribution bounds.

function $F$ of $x$ has the lower bound $L$ and the upper bound $U$. We denote this relation by $x \triangleleft T$; the family $\mathcal{X}$ may be written as $\{x \mid x \triangleleft T\}$.

Since a univariate random variable is determined by its cumulative distribution function, it seems appropriate to use the CDF for defining the $\checkmark \triangleleft$ '-relation. The reader however should be aware of the fact that this notion might not always conform with the intuition behind the concept of thickets. The following example was pointed out by the anonymous reviewer: Consider the random variable $x$ concentrated on the points 1 and 4 , with probability 0.5 each. Even if no probability is assigned to the interval $(2,3), x$ is represented by the thicket $\{((1,4), 0.5),((2,3), 0.5)\}$.

\subsection{Construction And Deconstruction of Distribution envelopes}

The graphs of the distribution envelopes $U$ and $L$ of a thicket $T$ can be constructed geometrically (Figure 1). In order to find the upper envelope $U$ we assume that the intervals $C_{i}$ are numbered so that $a_{i} \leq a_{i+1}$. We visualize the pairs $\left(C_{i}, p_{i}\right)$ by rectangles of height $p_{i}$, erected over the interval $C_{i}$. Beginning with $C_{1}$, we translate the rectangles $C_{i}$ in $y$-direction so that its bottom edge is at the height of the top edge of $C_{i-1}$. The left hand vertices of these rectangles define the graph of $U$. The lower distribution envelope $L$ is found by piling up the same rectangles after sorting them in ascending order by right hand interval borders $b_{i}$.

The inverse procedure can be used for deconstructing a pair of suitable distribution bounds into a thicket. Since we come back to this task in a more general context in Section 3, we will not go into details here. The interested reader can find a formal description of the deconstruction algorithm for univariate thickets in (Regan et al., 2004, Section 4). We only mention that the deconstruction of distribution bounds into a thicket is not necessarily unique (see Example 1), but always information preserving (i.e. is an invertible operation).

\subsection{UNIVARIATE DENV}

Distribution envelope determination (Berleant and Goodman-Strauss, 1998) is a method for bounding the result of a binary arithmetic operation " $\star$ " on random variables without making assumptions on their mutual dependence. As a prerequisite, the operation has to be extended to nonempty intervals. Then, 
the input random variables are discretized as thickets and DEnv computes distribution bounds on the resulting random variable, which is subsequently deconstructed into a thicket.

ALGORITHM 1. (Univariate DEnv).

Input: $\quad$ Two thickets $T^{\prime}=\left\{\left(C_{i}^{\prime}, p_{i}^{\prime}\right)\right\}, T^{\prime \prime}=\left\{\left(C_{j}^{\prime \prime}, p_{j}^{\prime \prime}\right)\right\}$ and a binary operation " $\star$ ", defined on $\mathbb{R}$ and extended to the case of nonempty open intervals as arguments.

Output: A thicket representing all random variables $x^{\prime} \star x^{\prime \prime}$ where $x^{\prime} \triangleleft T^{\prime}$ and $x^{\prime \prime} \triangleleft T^{\prime \prime}$. No assumption on the dependence of $x^{\prime}$ and $x^{\prime \prime}$ is made.

Step 1: Compute the intervals $C_{i j}=\left(a_{i j}, b_{i j}\right):=C_{i}^{\prime} \star C_{j}^{\prime \prime}$. The intervals $C_{i j}$ will be the thicket intervals, but their probability masses are still unknown.

Step 2: Compute the upper distribution bound $U$ by solving the optimization problem

$$
U(t)=\max \sum_{(i, j): a_{i j} \leq t} p_{i j},
$$

for all $t \in \mathbb{R}$, where the unknowns $p_{i j}$ are subject to the constraints

$$
\sum_{i} p_{i j}=p_{i}^{\prime}, \quad \sum_{j} p_{i j}=p_{j}^{\prime \prime} \quad \text { and } \quad 0 \leq p_{i j}
$$

Step 3: Compute the lower distribution bound $L$ of $T$ by solving the optimization problem

$$
L(t)=\min \sum_{(i, j): b_{i j} \leq t} p_{i j},
$$

for all $t \in \mathbb{R}$; where the unknowns $p_{i j}$ are subject to the constraints (3).

Step 4: Deconstruct $U$ and $L$ into the resulting thicket $T$.

Remark 1. The optimization problem in Steps 2 and 3 can be solved efficiently by linear programming, even for a large number of variables (Karloff, 1991). Since $U$ and $L$ are simple functions (piecewise constant with finite range), it is sufficient to compute the values of $L(t)$ and $U(t)$ at a finite number of suitably chosen arguments $t \in \mathbb{R}$. Hence the algorithm's implementation is straightforward once libraries for linear programming and interval arithmetic are available.

For the computation of $U$ one assumes a concentration of the probability mass $p_{i j}$ at $a_{i j}$; for the computation of $L$ one assumes a concentration at $b_{i j}$. This and the chosen optimization targets (minimum or maximum) ensure the correctness and pointwise optimality of the algorithm.

Dependence information formulated in terms of linear equalities or inequalities is easily incorporated in the univariate DEnv algorithm. An example 
of this is the Pearson correlation coefficient (Berleant and Zhang, 2004b). Furthermore, it is obvious how to extend the algorithm to operations with more than two operands.

\section{Multivariate thickets}

In this section we introduce multivariate thickets. Even if this concept is in some sense a straightforward generalization of the univariate case, there are also essential differences.

DEFINITION 2. A multivariate thicket is a finite set $T=\left\{\left(C_{i}, p_{i}\right)\right\}$ whose elements are pairs of nonempty open convex sets $C_{i} \subset \mathbb{R}^{d}$ and associated probability values $p_{i}$ such that $\sum p_{i}=1$.

DEFINITION 3. The lower and upper probability $\underline{p}, \bar{p}$ of a thicket $T=$ $\left\{\left(C_{i}, p_{i}\right)\right\}$ are the functions $\underline{p}$ and $\bar{p}$ defined on the sigma algebra $\mathcal{B}_{d}$ of Borel sets of $\mathbb{R}^{d}$ :

$$
\underline{p}: X \in \mathcal{B}_{d} \mapsto \sum_{C_{i} \subset X} p_{i}, \quad \bar{p}: X \in \mathcal{B}_{d} \mapsto \sum_{C_{i} \cap X \neq \varnothing} p_{i}
$$

Remark 2. The functions $\underline{p}$ and $\bar{p}$ satisfy the axioms of interval probability (Walley, 1996):

1. $\forall X \in \mathcal{B}_{d}: 0 \leq p(X) \leq \bar{p}(X) \leq 1$;

2. $\underline{p}\left(\mathbb{R}^{d}\right)=\bar{p}\left(\mathbb{R}^{d}\right)=1, \underline{p}(\varnothing)=\bar{p}(\varnothing)=0$;

3. $\bar{p}(X)+\underline{p}\left(\mathbb{R}^{d} \backslash X\right)=1$;

4. $\forall X, Y \in \mathcal{B}_{d}: X \cap Y=\varnothing \Longrightarrow$ $\underline{p}(X)+\underline{p}(Y) \leq \underline{p}(X \cup Y) \leq \underline{p}(X)+\bar{p}(Y) \leq \bar{p}(X \cup Y) \leq \bar{p}(X)+\bar{p}(Y)$.

DEFINITION 4. A random variable $x$ defined over $\mathcal{B}_{d}$ is represented by a thicket $T$, if $p(X) \leq P(x \in X) \leq \bar{p}(X)$ holds for all $X \in \mathcal{B}_{d}$. We denote this relation by $x \triangleleft T$. Two thickets $T_{1}, T_{2}$ are called equivalent if they represent the same set of random variables. If every random variable represented by $T_{1}$ is also represented by $T_{2}$, we call $T_{1}$ a subthicket of $T_{2}$.

Remark 3. In the univariate case, the $\triangleleft$-relation between random variables and thickets is defined via the thicket's distribution envelopes. There exists a multivariate generalization of distribution envelopes (see Section 3) but it cannot be used for an analogous definition. The reason for this will become clear in Example 2. 

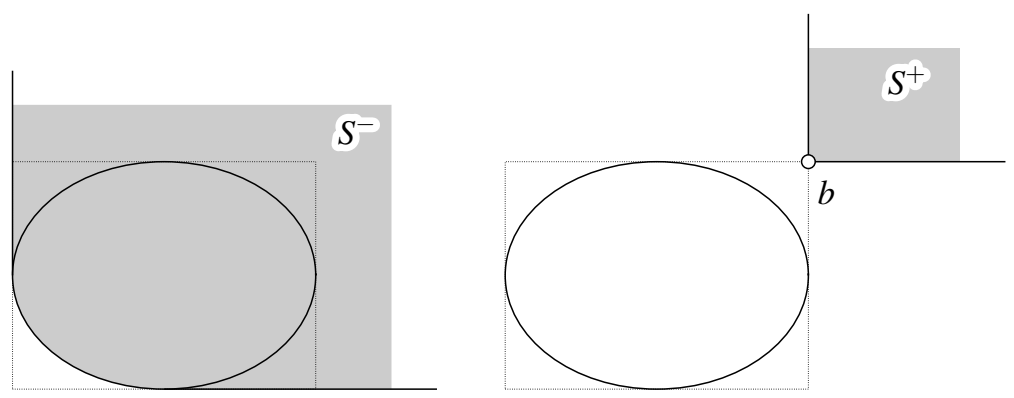

Figure 2. Definition of $S^{+}$and $S^{-}$.

Example 1. There is a trivial method for constructing a thicket equivalent to a given thicket $T=\left\{\left(C_{i}, p_{i}\right)\right\}$. We replace one thicket element $\left(C_{i}, p_{i}\right)$ by the two new elements $\left(C_{i}, \varepsilon\right),\left(C_{i}, p_{i}-\varepsilon\right)$ where $0<\varepsilon<p_{i}$. We call every thicket obtained from $T$ by a series of these operations a partition thicket of $T$. Clearly two thickets are equivalent if they have a common partition thicket. In this case we call them essentially identical. The converse is not true, i.e., the same family of random variables can be represented by essentially different thickets. An example of this is given by two equivalent thickets

$T_{1}=\{((0,2), 0.3),((1,3), 0.7)\}, T_{2}=\{((0,3), 0.3),((1,3), 0.4),((1,2), 0.3)\}$.

\subsection{Distribution EnVElopes}

DEFINITION 5. For an arbitrary subset $S$ of $\mathbb{R}^{d}$ we define $S^{+}$as the set of all points $\left(y_{1}, \ldots, y_{d}\right) \in \mathbb{R}^{d}$ such that $x_{i} \leq y_{i}$ holds for all points $\left(x_{1}, \ldots, x_{d}\right) \in S$ and all indices $i \in\{1, \ldots, d\}$. The set $S^{-}$is defined as $\bigcup_{x \in S}\{x\}^{+}$. When using coordinates with respect to a basis $A$ of $\mathbb{R}^{d}$, we write $S^{+}(A)$ and $S^{-}(A)$.

Examples of $S^{+}$and $S^{-}$are depicted in Figure 2. In $2 \mathrm{D}, S^{+}=b^{+}$where $b$ is the upper right corner of the bounding box.

DEFINITION 6. The upper and lower distribution envelope of a thicket $T=$ $\left\{\left(C_{i}, p_{i}\right)\right\}$ are the functions

$$
U_{T}: \mathbb{R}^{d} \rightarrow[0,1], t \mapsto \sum_{t \in C_{i}^{-}} p_{i}, \quad L_{T}: \mathbb{R}^{d} \rightarrow[0,1], t \mapsto \sum_{t \in C_{i}^{+}} p_{i} .
$$

When using coordinates with respect to the basis $A$ of $\mathbb{R}^{d}$, we write $L_{T}(A, t)$ and $U_{T}(A, t)$.

With the definitions $\left(u_{1}, \ldots, u_{d}\right) \leq\left(t_{1}, \ldots, t_{d}\right) \Longleftrightarrow u_{1} \leq t_{1}, \ldots, u_{d} \leq t_{d}$ and $t \leq:=\left\{u \in \mathbb{R}^{d} \mid u \leq t\right\}$, the MDF of $x$ is defined as $F: \mathbb{R}^{d} \rightarrow \mathbb{R}$, $F(t):=P(x \leq t)$, i.e., the probability assigned to the set $t \leq$. Definition 6 is the multivariate analogue to Definition 1. The definition of $L_{T}$ and $U_{T}$ 

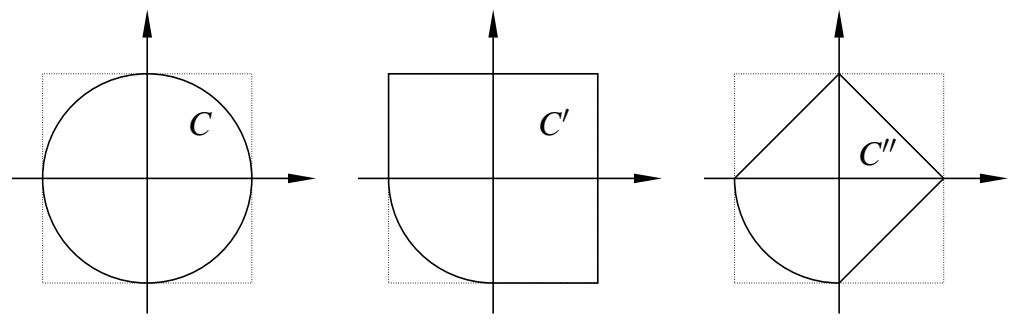

Figure 3. One-element thickets with identical distribution bounds.

ensures that they enclose the multivariate distribution function (MDF) of every random variable $x \triangleleft T$, regardless of the distribution of probability mass inside the convex sets $C_{i}$. For the definition of $U_{T}$, we assume a concentration of probability mass at points of the "lower left" border of $C_{i}$; for the definition of $L_{T}$ we assume a concentration at points with maximal coordinate values.

PROPOSITION 1. The multivariate distribution function $F$ of a random variable $x \triangleleft T$ has the property that $L_{T}(t) \leq F(t) \leq U_{T}(t)$ for all $t \in \mathbb{R}^{d}$.

Proof. The proof is a matter of combining the notions defined above (Definitions 3, 4 and 6): $x \triangleleft T$ implies that for all $t$ :

$$
F(t)=P(x \leq t) \leq \bar{p}\left(t^{\leq}\right)=\sum_{i:\left(C_{i} \cap t \leq\right) \neq \varnothing} p_{i}=\sum_{i: \exists u \in C_{i}: u \leq t} p_{i}=\sum_{i: t \in C_{i}^{-}} p_{i}=U_{T}(t)
$$

The inequality $F(t) \geq L_{T}(t)$ is shown in an analogous way.

The converse is not true, as demonstrated by the next example. Thus representing a thicket by distribution bounds loses information.

Example 2. Consider the one-element thickets $T=\{(C, 1)\}, T^{\prime}=\left\{\left(C^{\prime}, 1\right)\right\}$, and $T^{\prime \prime}=\left\{\left(C^{\prime \prime}, 1\right)\right\}$, where $C, C^{\prime}$ and $C^{\prime \prime}$ are bounded by arcs and straight lines as illustrated in Figure 3. Each thicket represents the random variables concentrated in the corresponding convex set, but the upper and lower distribution bounds $L$ and $U$ are the same for all three. $T^{\prime}$ represents precisely the random variables whose MDF lies between $L$ and $U$, and $C^{\prime \prime}$ is the smallest convex set such that the corresponding one-element thicket has these lower and upper distribution bounds. If the loss of information is measured in terms of the area, then $C^{\prime}$ is $20 \%$ larger than $C$ and $65 \%$ larger than $C^{\prime \prime}$.

\subsection{DeCONSTRUCtion OF DISTRIBUtion ENVELOPES}

The deconstruction of a pair $U, L$ of distribution envelopes into a thicket is a fundamental step of DEnv. The basic ideas are the same in both the univariate and the multivariate settings. We give a formal definition of the corresponding algorithm. 

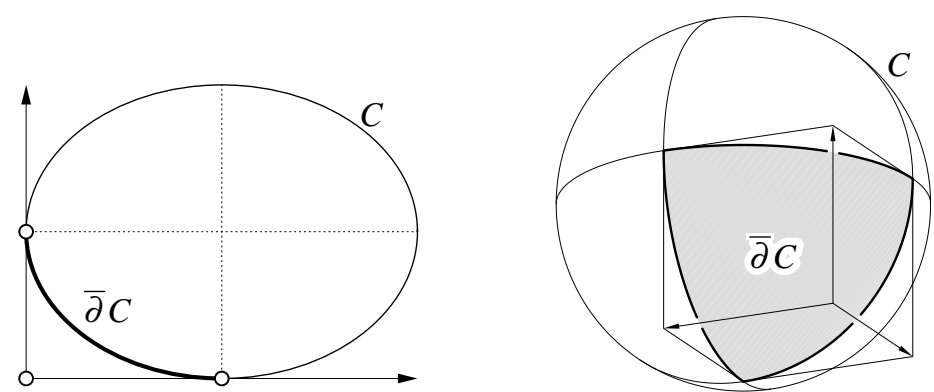

Figure 4. Definition of $\bar{\partial} C$ in $2 \mathrm{D}$ (left) and 3D (right).

DEFINITION 7. Assume that $F: \mathbb{R}^{d} \rightarrow[0,1]$ is a simple function with $\operatorname{range}(F)=\left\{f_{0}, \ldots, f_{n}\right\}$ such that $0=f_{0}<f_{1}<\ldots<f_{n}=1$. We consider the level sets $\left[F=f_{i}\right](i=0, \ldots, n)$, and for $z \in \mathbb{R}$ we define the level-type sets $[F \approx z]:=\overline{[F \geq z]} \cap \overline{[F<z]}$ (here $\bar{X}$ denotes topological closure of $X$ ).

DEFINITION 8. The bounding box of a subset $S \subset \mathbb{R}^{d}$ is the set $B B(S):=$ $\pi_{1}(S) \times \cdots \times \pi_{d}(S)$ where $\pi_{j}\left(x_{1}, \ldots, x_{d}\right):=x_{j}$ is the projection onto the $j$ th coordinate. If $C$ is a convex set with boundary $\partial C$, then $\bar{\partial} C$ is the set of all points $x \in \partial C$ which have a supporting hyperplane with exterior normal vector $n=\left(n_{1}, \ldots, n_{d}\right)$ such that $n_{1}, \ldots, n_{d} \leq 0$. Further we define $\eta C:=$ $C^{-} \cap B B(C)$. The convex hull of $M \subseteq \mathbb{R}^{d}$ is denoted by the symbol $C H(M)$.

The definition of $\bar{\partial} C$ is illustrated in Figure 4. Examples of $\eta C$ are found in Figure 3, where we have $C^{\prime}=\eta C=\eta C^{\prime \prime}$. Note that

$C$ open and convex $\Longrightarrow \mathrm{BB}(\eta C)=\mathrm{BB}(C),(\eta C)^{+}=C^{+},(\eta C)^{-}=C^{-}$.

These properties are used in the following algorithm, which is similar to the deconstruction algorithm for univariate thickets (Regan et al., 2004).

ALGORITHM 2. (Thicket deconstruction).

Input: Two simple functions $L, U$ with the properties $L(t) \leq U(t)$ for all $t \in \mathbb{R}^{d}, 0,1 \in \operatorname{range}(L) \cap \operatorname{range}(U), u \leq t \Longrightarrow L(u) \leq L(t), U(u) \leq$ $U(t)$ (i.e., both $L$ and $U$ are multivariate distribution functions).

Output: Thicket $T$ representing all random variables whose MDF $F$ fulfills $L \leq F \leq U$.

Step 1: Let $Z:=$ range $(L) \cup$ range $(U)$ and sort the members of $Z$ in ascending order: $0=z_{0}<\cdots<z_{n}=1$.

Step 2: For $i=1, \ldots, n$ let

$$
C_{i}=\eta C H\left(\bar{\partial} C H\left[U \approx z_{i}\right] \cup \bar{\partial} C H\left[L \approx z_{i}\right]\right)
$$

(see Figure 5) and define $p_{i}:=z_{i}-z_{i-1}$.

Step 3: Let $T=\left\{\left(C_{i}, p_{i}\right) \mid i=1, \ldots, n\right\}$. 




Figure 5. Constructing a thicket element $C_{i}$ (shaded area) from level sets of $U$ and $L$.

Proof (of correctness of Algorithm 2). We claim that for a random variable $x$ whose distribution function $F$ fulfills $L(t) \leq F(t) \leq U(t)$ for all $t \in \mathbb{R}^{d}$, we have $x \triangleleft T$. First we observe that

$$
C \subseteq C^{\prime} \Longrightarrow C^{+} \supseteq\left(C^{\prime}\right)^{+}, \eta C \subseteq \eta C^{\prime} ; \quad C_{i} \subseteq C_{i, L}:=\eta \mathrm{CH}\left(\bar{\partial} \mathrm{CH}\left[L \approx z_{i}\right]\right)
$$

The "lower left corners" of $[L \approx z]$ and $[U \approx z]$ determine the shape of these sets:

$$
(\mathrm{CH} \bar{\partial} \mathrm{CH}[U \approx z])^{-}=\mathrm{CH}[U \approx z], \quad t \in(\mathrm{CH} \bar{\partial} \mathrm{CH}[L \approx z])^{+} \Longrightarrow L(t) \geq z
$$

(cf. Figures 2 and 4). We aim at the inequality $L_{T}(t) \leq L(t)$ and use (11) to compute

$$
L_{T}(t)=\sum_{i: t \in C_{i}^{+}} p_{i} \leq \sum_{i: t \in C_{i, L}^{+}} p_{i}=\sum_{i: t \in C_{i, L}^{+}} p_{i}
$$

By (9) and (12), $t \in C_{i, L}^{+}$implies that $L(t) \geq z_{i}$. Thus

$$
L_{T}(t) \leq \sum_{i: L(t) \geq z_{i}}\left(z_{i}-z_{i-1}\right)=\max \left\{z_{j} \mid L(t) \geq z_{j}\right\} \leq L(t)
$$

As to $U_{T}$, we define $C_{i, U}$ analogous to (11) and observe $L \leq U \Longrightarrow C_{i, L}^{-} \subseteq$ $C_{i, U}^{-}$:

$$
\begin{aligned}
U_{T}(t) & =\sum_{i: t \in C_{i}^{-}} p_{i}=\sum_{i: t \in C_{i, U}^{-}} p_{i}=\sum_{i: t \in \mathrm{CH}\left[U \approx z_{i}\right]} p_{i} \\
& \geq \sum_{i: t \in\left[U \approx z_{i}\right]} p_{i}=\max \left\{z_{i} \mid t \in\left[U \geq z_{i}\right]\right\}=U(t) .
\end{aligned}
$$

With $L_{T} \leq L$ and $U \leq U_{T}$ as shown above and the assumption $L \leq F \leq U$ as required, we have shown $L_{T} \leq F \leq U_{T}$, i.e., $x \triangleleft T$.

In general a thicket $T_{0}$ is only a subthicket (c.f. Definition 4 ) of the thicket $T$ constructed by Algorithm 2 from $U_{T_{0}}$ and $L_{T_{0}}$, i.e., $x \triangleleft T$ does not imply $x \triangleleft T_{0}$. This is in contrast to the univariate case.

DEFINITION 9. If finite sets $Z \subseteq Z^{\prime} \subset \mathbb{R}$ have the property that $\min Z=$ $\min Z^{\prime}$ and $\max Z=\max Z^{\prime}$, then $Z^{\prime}$ is called a partition of $Z$. 
If the set $Z$ in Step 1 of Algorithm 2 is replaced by a partition $Z^{\prime}$, the output thicket will be a partition thicket of the original one. Now we have provided all tools to formulate the multivariate version of the DEnv algorithm.

ALGORITHM 3. (Multivariate DEnv).

Input: $\quad$ Two thickets $T^{\prime}=\left\{\left(C_{i}^{\prime}, p_{j}^{\prime}\right)\right\}, T^{\prime \prime}=\left\{\left(C_{i}^{\prime \prime}, p_{i}^{\prime \prime}\right)\right\}$ and a binary operation " $\star$ ", defined on $\mathbb{R}^{d}$ and extended to convex sets in the sense that $t \in C, t^{\prime} \in C^{\prime} \Longrightarrow t \star t^{\prime} \in C \star C^{\prime}$ and $C \star C^{\prime}$ is convex.

Output: A thicket representing all random variables $x^{\prime} \star x^{\prime \prime}$ where $x^{\prime} \triangleleft T^{\prime}$ and $x^{\prime \prime} \triangleleft T^{\prime \prime}$. No assumption on the dependence of $x^{\prime}$ and $x^{\prime \prime}$ is made.

Step 1: Compute $C_{i j}=C_{i}^{\prime} \star C_{j}^{\prime \prime}$.

Step 2: Compute the upper distribution bound $U$ by solving the optimization problem

$$
U(t):=\max \sum_{t \in C_{i j}^{-}} p_{i j},
$$

for each $t \in \mathbb{R}$, where the unknowns $p_{i j}$ are subject to the constraints (3).

Step 3: Compute the lower distribution bound $L$ of $T$ by solving the optimization problem

$$
L(t):=\min \sum_{t \in C_{i j}^{+}} p_{i j} .
$$

for each $x \in \mathbb{R}$, where the unknowns $p_{i j}$ are subject to the constraints (3).

Step 4: Deconstruct $U$ and $L$ into the resulting thicket $T$.

Proof (of correctness of Algorithm 3). The function $U$ in (16) is an upper bound of the MDF of all random variables $x=x^{\prime} \star x^{\prime \prime}$ because the level-type sets $\left[U \approx z_{i}\right]$ enclose the corresponding level sets of the MDF of $x$ and the corresponding function values are maximal, subject to the necessary constraint (3). Analogously it follows that $L$ is a lower bound of the MDF of $x$.

The basic steps of this algorithm are similar to the univariate DEnv algorithm (Algorithm 1). However, the result needs a different interpretation:

- The multivariate DEnv algorithm is information losing. This is due to the information losing intermediate representation of a thicket by its distribution bounds.

- The output of multivariate DEnv depends on the coordinate frame.

Both issues are drawbacks for the use of DEnv in geometric constructions. The loss of information in the DEnv algorithm can be reduced by a certain amount if we choose an appropriate basis of $\mathbb{R}^{d}$. In higher dimensions it apparently is not easy to minimize $\int(U-L)^{2}$ or a similar target functional over all possible orthonormal bases of $\mathbb{R}^{d}$ (in case we work in Euclidean geometry). Therefore we present an improved version of multivariate DEnv in the next section. The main idea is to combine DEnv results that were obtained with respect to different coordinate frames of $\mathbb{R}^{d}$. 


\section{Nested thickets and thicket intersection}

In this section we introduce nested thickets and thicket intersection in order to reduce the shortcomings of the multivariate DEnv algorithm.

DEFINITION 10. A thicket $T=\left\{\left(C_{i}, p_{i}\right) \mid i=0, \ldots, n\right\}$ is called nested if there exists a permutation $\pi$ of $\{0, \ldots, n\}$ such that $C_{\pi(i+1)} \subset C_{\pi(i)}$.

We tacitly assume that for a nested thicket $\left\{\left(C_{i}, p_{i}\right)\right\}$ the sets $C_{i}$ are numbered such that $C_{i+1} \subseteq C_{i}$. The lower and upper probability of a nested thicket are of simple shape. If $X \in \mathcal{B}_{d}$, we have

$$
\underline{p}(X)=\sum_{i=\underline{m}}^{n} p_{i}, \quad \bar{p}(X)=\sum_{i=0}^{\bar{m}} p_{i}
$$

where $\underline{m}:=\min \left\{i \mid X \subset C_{i}\right\}$ and $\bar{m}:=\max \left\{i \mid X \cap C_{i} \neq \varnothing\right\}$. In particular this implies

$$
P\left(x \in C_{i}\right) \geq \sum_{j=i}^{n} p_{j} \quad \text { and } \quad P\left(x \in \mathbb{R}^{d} \backslash C_{i}\right) \leq \sum_{j=0}^{i-1} p_{j} .
$$

DEFINITION 11. The thicket $T$ is the intersection of the thickets $T_{1}$ and $T_{2}$ if

$$
x \triangleleft T \Longleftrightarrow\left(x \triangleleft T_{1}\right) \text { and }\left(x \triangleleft T_{2}\right) .
$$

The intersection of $T_{1}$ and $T_{2}$ is denoted by $T_{1} \cap T_{2}$.

Note that the intersection of two thickets does not always exist but univariate thickets $T_{1}, T_{2}$ can always be intersected: If $U_{i}$ and $L_{i}$ are the distribution envelopes of $T_{i}$, we can deconstruct $T=T_{1} \cap T_{2}$ from its distribution envelopes

$$
U:=\min \left\{U_{1}, U_{2}\right\}, \quad L:=\max \left\{L_{1}, L_{2}\right\} .
$$

In the same way it is possible to intersect multivariate thickets but the result will usually be only a superset of intersection. Note further that the distribution bounds $U_{i}$ and $L_{i}$ have to refer to the same basis of $\mathbb{R}^{d}$.

In contrast to general multivariate thickets, the intersection of nested thickets exists. The intersection algorithm is based on the representation of nested thickets via nested stacks:

DEFINITION 12. For $i=0, \ldots, n$ assume that $B_{i} \subset \mathbb{R}^{d+1}$ is the cylinder $C_{i} \times\left(0, h_{i}\right)$ with a nonempty convex domain $C_{i}$ as basis. If $C_{i+1} \subseteq C_{i}$ and $h_{i+1} \geq h_{i}$, the union $\bigcup_{i=0}^{n} C_{i}$ is called a nested stack.

For a nested thicket $T=\left\{\left(C_{i}, p_{i}\right) \mid i=0, \ldots, n\right\}$ we may construct the nested stack defined by cylinders $B_{i}=C_{i} \times\left(0, h_{i}\right)$ with $h_{i}=\sum_{j=0}^{i} p_{j}$. Conversely a nested stack defined by cylinders $C_{i} \times\left(0, h_{i}\right)$ can be deconstructed into a nested thicket $\left\{\left(C_{i}, p_{i}\right) \mid i=0, \ldots, n\right\}$ by recursively defining $p_{0}=h_{0}$ and $p_{i}=h_{i}-h_{i-1}$ for $i>0$. The deconstruction of the nested stack into a thicket does not necessarily reproduce the original thicket, but at least an essentially identical thicket (cf. Example 1). Therefore we can represent nested thickets by nested stacks and vice versa. 
LEMMA 1. The intersection of the two thickets $T_{1}=\left\{\left(C_{1}, 1\right)\right\}$ and $T_{2}=$ $\left\{\left(C_{2}, 1\right)\right\}$ is the thicket $T_{3}=\left\{\left(C_{3}, 1\right)\right\}$ with $C_{3}=C_{1} \cap C_{2}$.

Proof. The case $C_{1}=C_{2}$ being trivial, we assume $C_{1} \neq C_{2}$. Since $T_{3}$ is a subthicket of $T_{1}$ and $T_{2}$, every random variable represented by $T_{3}$ is also represented by $T_{1}$ and $T_{2}$. We have to show the converse. Assume that $x \triangleleft T_{1}$, $x \triangleleft T_{2}$, but $x \nless T_{3}$. We denote the lower and upper probabilities of $T_{i}$ by $\underline{p}_{i}$ and $\bar{p}_{i}$. There exists $X \in \mathcal{B}_{d}$ such that $\underline{p}_{i}(X) \leq P(x \in X) \leq \bar{p}_{i}(X)$ for $i=1, \underline{\underline{p}}$ and either $p_{3}(X)>P(x \in X)$ (case 1$)^{-i}$ or $\bar{p}_{3}(X)<P(x \in X)$ (case 2), where $P(x \in X)$ is the probability of $x$ being contained in $X$.

In case $1, x \triangleleft T_{2}$ and $\underline{p}_{2}\left(C_{1} \backslash C_{2}\right)=\bar{p}_{2}\left(C_{1} \backslash C_{2}\right)=0$ imply $P\left(x \in C_{1} \backslash C_{2}\right)=$ 0 , and $x \triangleleft T_{1}, \underline{p}_{1}\left(C_{1}\right)=1$ imply $P\left(x \in C_{1}\right)=1$. Thus

$$
P\left(x \in C_{3}\right)=P\left(x \in C_{1} \backslash C_{2}\right)+P\left(x \in C_{3}\right)=P\left(x \in C_{1}\right)=1
$$

which leads to the contradiction $1 \geq \underline{p}_{3}(X)>P(x \in X) \geq P\left(x \in C_{3}\right)=1$.

Case 2 is possible only if $X \cap C_{3} \stackrel{=}{=} \varnothing$ but $X \cap C_{1} \neq \varnothing$ and $X \cap C_{2} \neq \varnothing$. This implies the contradiction

$0=\bar{p}_{3}(X)<P(x \in X)=P\left(x \in X \cap C_{1}\right)+P\left(x \in X \backslash C_{1}\right) \leq P\left(x \in C_{1} \backslash C_{2}\right)=0$

and finishes the proof.

LEMMA 2. The intersection of the thickets $T_{i}=\left\{\left(\mathbb{R}^{d}, p\right),\left(C_{i}, 1-p\right)\right\}(i=$ $1,2)$ with $0<p<1$ is the thicket $T_{3}=\left\{\left(\mathbb{R}^{d}, p\right)\right.$, $\left.\left(C_{3}, 1-p\right)\right\}$, where $C_{3}=$ $C_{1} \cap C_{2}$.

Proof. The random variable $x^{\prime}=\max \{0,(x-p) /(1-p)\}$ is represented by the thicket $T_{i}^{\prime}=\left\{\left(C_{i}, 1\right)\right\}$ if and only if $x \triangleleft T_{i}$. Therefore, the result follows from Lemma 1.

THEOREM 1. Assume that the nested thicket $T_{i}=\left\{\left(C_{i j}, p_{i j}\right) \mid j=0, \ldots, n_{i}\right\}$ $(i=1,2)$ is represented by the nested stack $B_{i}$. Then the intersection of $T_{1}$ and $T_{2}$ is represented by the nested stack $B_{1} \cap B_{2}$.

Proof. Without loss of generality we assume $n_{1}=n_{2}=: n$ and $p_{1 j}=p_{2 j}=$ : $p_{j}$. This condition can always be fulfilled if we replace $T_{1}$ and $T_{2}$ by suitable partition thickets. The set $B_{3}:=B_{1} \cap B_{2}$ is a nested stack and corresponds to a thicket $T_{3}=\left\{\left(C_{3 j}, p_{j}\right) \mid j=0, \ldots, n\right\}$, where $C_{3 j}=C_{1 j} \cap C_{2 j} . T_{3}$ is a subthicket of $T_{1}$ and $T_{2}$, i.e., every random variable represented by $T_{3}$ is also represented by $T_{1}$ and $T_{2}$. We have to show the converse.

We assume that this is not the case: There exists a random variable with $x \triangleleft T_{1}$ and $x \triangleleft T_{2}$ but $x \Varangle T_{3}$. If $\underline{p}_{i}$ and $\bar{p}_{i}$ denote the lower and upper probability of $T_{i}$, there exists a set $X^{-i} \in \mathcal{B}_{d}$ such that $p_{3}(X)>P(x \in X)$ or $\bar{p}_{3}(X)<P(x \in X)$. We consider the case $\underline{p}_{3}(X)>P(x \in \mathcal{\in})$ first. Nestedness of the thickets $T_{i}$ implies

$$
\underline{p}_{i}(X)=\sum_{C_{i j} \subset X} p_{j}=\sum_{j=\underline{m}_{i}}^{n} p_{j}
$$

where

$$
\underline{m}_{i}:=\min \left\{j \mid C_{i j} \subset X\right\}, \quad i=1,2,3 \text {. }
$$


Hence $\underline{m}_{3}<\min \left\{\underline{m}_{1}, \underline{m}_{2}\right\}$, i.e., $C_{3 \underline{m}_{3}} \subset X$ but neither $C_{\underline{1}_{m_{3}}} \subset X$ nor $C_{2 \underline{m}_{3}} \subset$ $X$. We let $T_{i}^{\prime}=\left\{\left(C_{i 0}^{\prime}, p_{0}^{\prime}\right),\left(C_{i 1}^{\prime}, p_{1}^{\prime}\right)\right\}$ where

$$
C_{i 0}^{\prime}=\mathbb{R}^{d}, \quad C_{i 1}^{\prime}=C_{i \underline{m}_{3}}, \quad p_{0}^{\prime}=\sum_{i=0}^{\underline{m}_{3}-1} p_{i}, \quad p_{1}^{\prime}=1-p_{0}^{\prime} .
$$

and denote the corresponding lower and upper probabilities by $p_{i}^{\prime}$ and $\bar{p}_{i}^{\prime}$. Because $T_{i}$ is a subthicket of $T_{i}^{\prime}, x$ is also represented by $T_{1}^{\prime}$ and $T_{2}^{\prime}$. Because of $\underline{p}_{3}^{\prime}(X)=\underline{p}(X)>P(x \in X), x$ is not represented by $T_{3}^{\prime}$. This is a contradiction to Lemma 2 . Now we consider the case $\bar{p}(X)<P(x \in X)$. The proof is similar to the previous case. We have

$$
\bar{p}_{i}(X)=\sum_{C_{i j} \cap X \neq \varnothing} p_{j}=\sum_{j=0}^{\bar{m}_{i}},
$$

where

$$
\bar{m}_{i}:=\max \left\{j \mid C_{i j} \cap X \neq \varnothing\right\}, \quad i=1,2,3 .
$$

Hence $\bar{m}_{3}>\max \left\{\bar{m}_{1}, \bar{m}_{2}\right\}$, i.e., $C_{3 \bar{m}_{3}} \cap X \neq \varnothing$ but $C_{1 \bar{m}_{3}} \cap X=\varnothing$ and $C_{2 \bar{m}_{3}} \cap X=\varnothing$. We let $T_{i}^{\prime \prime}=\left\{\left(C_{i 0}^{\prime \prime}, p_{0}^{\prime \prime}\right),\left(C_{i 1}^{\prime \prime}, p_{1}^{\prime \prime}\right)\right\}$, where

$$
C_{i 0}^{\prime \prime}=\mathbb{R}^{d}, \quad C_{i 1}^{\prime \prime}=C_{i \bar{m}_{3}}, \quad p_{0}^{\prime \prime}=\sum_{i=0}^{\bar{m}_{3}-1} p_{i}, \quad p_{1}^{\prime \prime}=1-p_{0}^{\prime \prime} .
$$

and denote the corresponding lower and upper probabilities by $p_{i}^{\prime \prime}$ and $\bar{p}_{i}^{\prime \prime}$. Because $T_{i}$ is a subthicket of $T_{i}^{\prime \prime}, x$ is also represented by $T_{1}^{\prime \prime}$ and ${\overline{T_{2}^{\prime \prime}}}^{\prime}$. Because of $\bar{p}_{3}^{\prime \prime}(X)=\bar{p}(X)>P(x \in X), x$ is not represented by $T_{3}^{\prime \prime}$. Again, this is a contradiction to Lemma 2.

Example 3. (Nested thicket intersection) The intersection of the nested thickets

$$
T_{1}=\{((1,6), 0.5),((3,4), 0.5)\}, \quad T_{2}=\{((0,7), 0.3),((2,5), 0.7)\}
$$

is the nested thicket $T_{1} \cap T_{2}=\{((1,6), 0.3),((2,5), 0.2),((3,4), 0.5)\}$.

In order to intersect arbitrary thickets, we have to transform them to nested thickets. We cannot expect that to be possible without losing information:

ALGORITHM 4. (Nested thicket conversion).

Input: $\quad$ Thicket $T=\left\{\left(C_{i}, p_{i}\right)\right\}$.

Output: Nested thicket $T^{\prime}$ of which $T$ is a subthicket.

Step 1: Denote by $U_{T}$ and $L_{T}$ the distribution envelopes of $T$. Let $Z$ be the union of range $\left(U_{T}\right)$ and range $\left(1-L_{T}\right)$ and sort its elements in ascending order: $0=z_{0}<\cdots<z_{n}=1$. If desired, $Z$ can be replaced by a partition of $Z$.

Step 2: For $k=0, \ldots, n-1$ let $u_{k}$ be the largest value in range $\left(U_{T}\right)$ that is not larger than $z_{k}$ and let $l_{k}$ be the smallest value in range $\left(1-L_{T}\right)$ that is not larger than $z_{k}$.

Step 3: Let

$$
C_{k}^{\prime}=\eta C H\left(\bar{\partial}\left[U_{T} \approx u_{k}\right] \cap \bar{\partial}\left[L_{T} \approx 1-l_{k}\right]\right) .
$$

If $k=n-1$ or if $C_{k}^{\prime}$ is empty, let $k_{0}:=k$ and continue with the next step. 

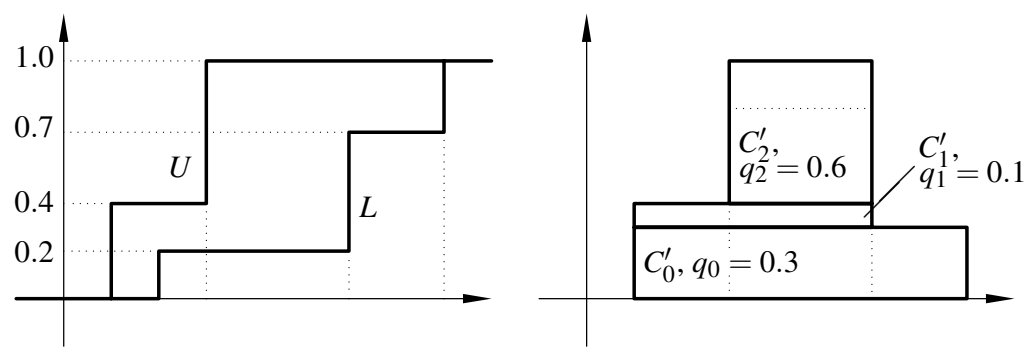

Figure 6. Nested thicket representation.

Step 4: For $k=0, \ldots, k_{0}-2$ let $p_{k}^{\prime}=z_{k+1}-z_{k}$ and let $p_{k_{0}-1}^{\prime}=1-\sum_{l=0}^{k_{0}-2} p_{l}^{\prime}$. Step 5: The output of the algorithm is the thicket $T^{\prime}=\left\{\left(C_{i}^{\prime}, p_{i}^{\prime}\right) \mid i=\right.$ $\left.0, \ldots, k_{0}-1\right\}$.

Proof (of correctness of Algorithm 4). The thicket $T^{\prime}$ is well defined because $C_{0}^{\prime}$ is never empty. It is no loss of generality to assume range $\left(U_{T}\right)=\operatorname{range}(1-$ $\left.L_{T}\right)$. If this condition is not fulfilled, we can replace $T$ by a suitable partition thicket. By construction, the thicket $T^{\prime}$ is nested and there exists an injection $\iota_{1}:\left\{0, \ldots, k_{0}-1\right\} \rightarrow\{0, \ldots, n\}$ such that $C_{i}^{\prime+}=C_{\iota_{1}(i)}^{+}$and, for $i \neq k_{0}-1, p_{i}^{\prime}=$ $p_{\iota_{1}(i)}$. Furthermore, $p_{k_{0}-1}^{\prime}$ is the sum over all values $p_{i}$ where $i \notin \operatorname{range}\left(\iota_{1}\right)$. This implies $L_{T^{\prime}} \leq L_{T}$.

Analogously, there exists an injection $\iota_{2}:\left\{0, \ldots, k_{0}-1\right\} \rightarrow\{0, \ldots, n\}$ such that $C^{\prime-}{ }_{i}=C_{\iota_{2}(i)}^{-}$and, for $i \neq k_{0}-1, p_{i}^{\prime}=p_{\iota_{2}(i)}$. Again $p_{k_{0}-1}^{\prime}$ is the sum over all values $p_{i}$ where $i \notin$ range $\left(\iota_{2}\right)$. This implies $U_{T} \leq U_{T^{\prime}}$ and $T$ is a subthicket of $T^{\prime}$.

Example 4. We convert the univariate thicket $T$ represented by the distribution envelopes $U, L$ of Figure 6 , left, into a nested thicket. First we unite the ranges of $U$ and $1-L$ and obtain the set $Z=\{0.0,0.3,0.4,0.8,1.0\}$. Now we construct the sets $C_{0}^{\prime}, C_{1}^{\prime}$ and $C_{2}^{\prime}$ according to Step 2 of the nested thicket conversion algorithm. Since $C_{3}^{\prime}$ is empty, we leave Step 3 with $k_{0}=2$ and assign the complete remaining probability mass 0.2 to $C_{2}^{\prime}$.

Nested thicket conversion with Algorithm 4 works for all thickets. The procedure however is information losing and depends on the coordinate frame. In fact, there are two information leaks. One is due to the intermediate representation of a thicket by distribution envelopes. The second potential loss of information occurs if we leave Step 3 with $k_{0}<n-1$. In this case, we have to assign the remaining probability mass to the set $C_{k_{0}}^{\prime}$ which means extending both distribution bounds.

The thicket of Example 4 cannot be converted into an equivalent nested thicket while the thicket $T_{1}$ of Equation (6) can. The equivalent nested thicket is the thicket $T_{2}$ of (6).

The main reason for using nested thickets in multivariate DEnv is the possibility to combine the results DEnv with respect to different coordinate frames by nested thicket intersection. 
ALGORITHM 5. (Multivariate DEnv with intersection).

Input: $\quad$ Two thickets $T^{\prime}=\left\{\left(C_{i}^{\prime}, p_{j}^{\prime}\right)\right\}, T^{\prime \prime}=\left\{\left(C_{i}^{\prime \prime}, p_{i}^{\prime \prime}\right)\right\}$ and a binary operation " $\star$ ", defined on $\mathbb{R}^{d}$ and extended to nonempty open convex sets.

Output: A thicket representing all random variables $x^{\prime} \star x^{\prime \prime}$ where $x^{\prime} \triangleleft T^{\prime}$ and $x^{\prime \prime} \triangleleft T^{\prime \prime}$. No assumption on the dependence of $x^{\prime}$ and $x^{\prime \prime}$ is made.

Step 1: Choose a certain number of bases $A_{i}$ of $\mathbb{R}^{d}$. For each of them perform the multivariate DEnv algorithm (Algorithm 3). Denote the resulting thickets by $T_{i}$.

Step 2: Use nested thicket conversion (Algorithm 4) to transform the thicket $T_{i}$ to a nested thicket $T_{i}^{\prime}$. Use the basis $A_{i}$ for this transformation.

Step 3: Return the thicket $T=\bigcap T_{i}^{\prime}$.

Proof (of correctness of Algorithm 5). The algorithm is correct because the thickets $T_{i}$ represent every random variable $x^{\prime} \star x^{\prime \prime}$ if $x^{\prime} \triangleleft T^{\prime}$ and $x^{\prime \prime} \triangleleft T^{\prime \prime}$, and the nested thicket transformation produces thickets $T_{i}^{\prime}$ with the same property. According to Theorem 1 this is also true for the output thicket $T$.

Remark 4. In an actual implementation of the algorithm it is not necessary to deconstruct the distribution bounds $U_{i}, L_{i}$ into thickets $T_{i}$ in Step 1 , since we need precisely these distribution bounds (and not the thickets themselves) for the nested thicket conversion in Step 2.

Remark 5. The applications one has in mind should determine which bases to choose in Step 1 of the above algorithm. Probably it is often sufficient to choose $2^{d}$ bases $A_{i}$ so that $\bigcup 0^{+}\left(A_{i}\right)=\mathbb{R}^{d}$. In $2 \mathrm{D}$, these could be for example

$$
A_{0}=\left(\begin{array}{ll}
1 & 0 \\
0 & 1
\end{array}\right), \quad A_{1}=\left(\begin{array}{rr}
0 & 1 \\
-1 & 0
\end{array}\right), \quad A_{2}=\left(\begin{array}{rr}
-1 & 0 \\
0 & -1
\end{array}\right), \quad A_{3}=\left(\begin{array}{rr}
0 & -1 \\
1 & 0
\end{array}\right) .
$$

We use the bases (32) in the example of Section 5 .

The multivariate DEnv with intersection reduces the loss of information due to intermediate thicket representation by distribution envelopes. It induces, however, a loss of information in conversion to nested thickets. What is worse depends on the particular examples. Certain families of random variables can be represented very well by nested thickets while others cannot. Some arguments in favor of the refined second version of multivariate DEnv are:

- The loss of information due to thicket representation by distribution envelopes might be considerable (Example 2) while the nested thicket conversion is often information preserving or nearly information preserving.

- In geometric constructions with imprecise input data (as well as in interval arithmetic) the excessive growth of tolerance regions is a problem. The multivariate DEnv algorithm with intersection allows better control of the size of the convex sets $C_{i}$. 


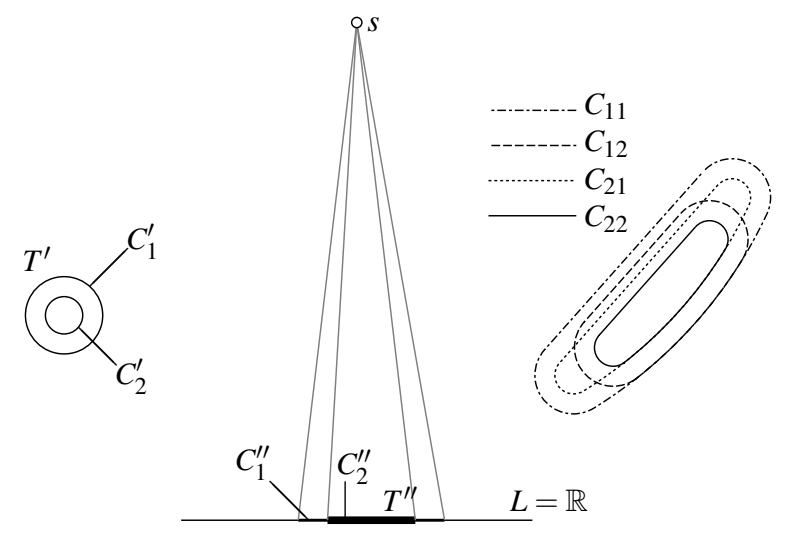

Figure 7. Reflection of a point in a line.

- The refined multivariate DEnv algorithm at least approaches geometric invariance.

\section{An Example}

This section provides a comprehensive example illustrating the algorithms of this article.

\subsection{Definition OF A BINARY OPERATION}

In the Euclidean plane we are given a straight line $L$ and a point $s \notin L$. The straight line $L$ is identified with $\mathbb{R}$; every point $r \in L$ corresponds to a real number and vice versa. We use $s$ and $L$ to define a binary operation $\star: \mathbb{R}^{2} \times \mathbb{R} \rightarrow \mathbb{R}^{2}$. The point $a \star r$ is defined as the reflection image of the point $a$ in the straight line through $s$ and $r \in L$.

In Figure 7 this situation is visualized. We anticipatorily assume that both $a$ and $r$ are random variables, represented by thickets $T^{\prime}$ and $T^{\prime \prime}$, respectively.

\subsection{Extension to INTERVALS}

We have to extend "“" to convex subsets of $\mathbb{R}^{2}$ and $\mathbb{R}$ : All points of a convex set $A$ are to be reflected in all straight lines $S$ that contain the point $s$ and intersect $L$ in an interval $R$. The set $M$ that is swept by this one-parameter family of reflected copies of $A$ is described in (Hu and Wallner, 2004). It is bounded by parts of the boundary of the reflection of $A$ in the two lines through the boundary points of $R$ and two circular arc segments with center $s$. Since our algorithm requires convex sets as result of the operation " $\star$ ", we replace $M$ by its convex hull $\mathrm{CH}(M)$, i.e., the extension of "*" to convex sets is defined by $A \star R:=\mathrm{CH}(M)$. 


\subsection{REPRESENTATION BY THICKETS}

Now we meet the requirements for an extension of " $\star$ " to multivariate thickets. We consider a concrete example. The position of the point $a$ as well as the intersection of the straight line $S$ with $L$ are random variables represented by thickets $T^{\prime}$ and $T^{\prime \prime}$, respectively. We choose $T^{\prime}=\left\{\left(C_{1}^{\prime}, 0.6\right),\left(C_{2}^{\prime}, 0.4\right)\right\}$, where $C_{1}^{\prime}$ and $C_{2}^{\prime}$ are the concentric disks shown by Figure 7 . Further, we let $T^{\prime \prime}=\left\{\left(C_{1}^{\prime \prime}, 0.8\right),\left(C_{2}^{\prime \prime}, 0.2\right)\right\}$, where $C_{1}^{\prime \prime}$ and $C_{2}^{\prime \prime}$ are the two intervals indicated in Figure 7.

\subsection{UPPER AND LOWER DISTRIBUTION ENVELOPES}

The first step in multivariate DEnv with intersection consists of computing the convex sets $C_{i j}=C_{i}^{\prime} \star C_{j}^{\prime \prime}$. In our example, each $C_{i j}$ is bounded by three circular arcs and one straight line segment (see Figure 7). We distinguish the different $C_{i j}^{\prime} s$ by line style.

The next step is the computation of distribution envelopes with respect to different bases of $\mathbb{R}^{2}$. We choose the initial basis $A_{0}$ of Equation (32) and compute the corresponding distribution envelopes $U_{0}=U_{0}\left(A_{0}\right)$ and $L_{0}=$ $L_{0}\left(A_{0}\right)$ :

Both upper and lower distribution envelope are simple functions of finite range. The value $U_{0}(t)$ is defined as the solution of the optimization problem

$$
U_{0}(t)=\max \sum_{t \in C_{i j}^{-}} p_{i j} .
$$

The variables to solve for are the unknown probability values $p_{i j}$ of $C_{i j}$; the necessary constraints are

$p_{11}+p_{12}=0.6, \quad p_{21}+p_{22}=0.4, \quad p_{11}+p_{21}=0.8, \quad p_{12}+p_{22}=0.2, \quad p_{i j} \geq 0$.

Figure 8 shows that $\mathbb{R}^{2}$ can be divided into five regions such that the target functional (33) is the same for all points of one region. Hence the range of $U_{0}$ is found by solving five optimization problems, two of which are trivial: the empty sum yields zero and the sum over all values $p_{i j}$ yields one. The three remaining target functionals and the corresponding solutions, found by linear programming, are

$$
0.6=\max \left\{p_{11}\right\}, \quad 0.8=\max \left\{p_{11}+p_{12}\right\}, \quad 1.0=\max \left\{p_{11}+p_{12}+p_{21}\right\} .
$$

Hence the range of $U_{0}$ is the set $\{0.0,0.6,0.8,1.0\}$.

The range of $L_{0}$ is computed in a similar way. We have to solve five minimization problems. The three nontrivial solutions are

$$
0.0=\min \left\{p_{22}\right\}, \quad 0.2=\min \left\{p_{22}+p_{12}\right\}, \quad 0.4=\min \left\{p_{22}+p_{12}+p_{21}\right\}
$$

and the range of $L_{0}$ is $\{0.0,0.2,0.4,1.0\}$.

Both $U_{0}$ and $L_{0}$ are discontinuous on points of three curves shown in bold by Figure 8 . These lines of discontinuity are composed of parts of the convex sets $C_{i j}$ and half lines parallel to the coordinate directions of $A_{0}$. 

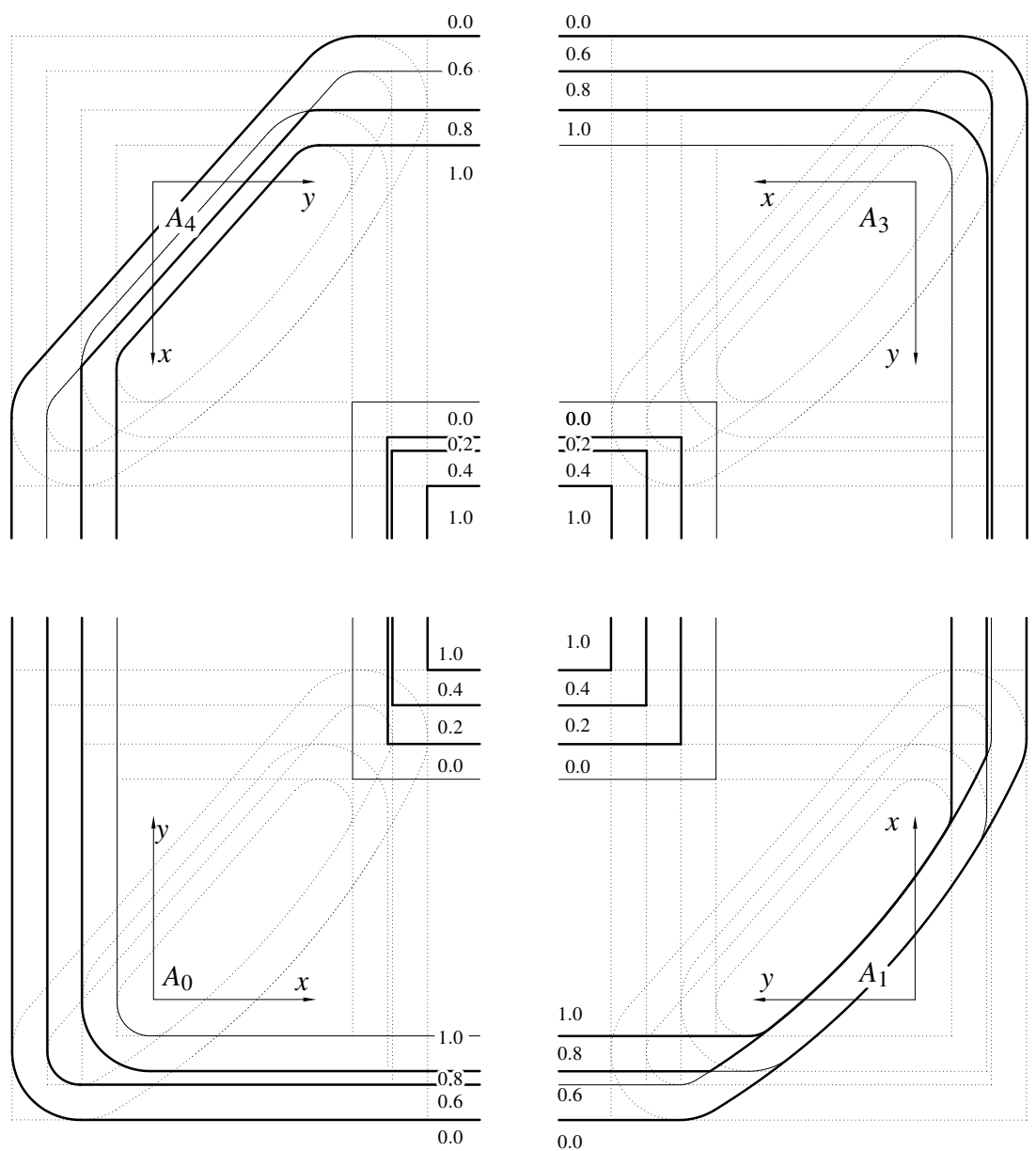

Figure 8. Distribution envelopes with respect to different coordinate frames.

We repeat the above steps for $i=1,2,3$ in order to compute distribution bounds $U_{i}, L_{i}$ with respect to the remaining bases $A_{i}$ of (32). The target functionals to be minimized are always those of (36). The minimal values are the function values of the lower distribution bounds. The target functionals to be maximized yield the function values of the upper distribution bounds. These functionals are the same for $U_{0}$ and $U_{3}$. For $U_{1}$ and $U_{2}$ they are

$$
\begin{aligned}
0.6 & =\max \left\{p_{11}\right\}, & 0.8 & =\max \left\{p_{11}+p_{12}\right\} \\
0.8 & =\max \left\{p_{11}+p_{21}\right\}, & 1.0 & =\max \left\{p_{11}+p_{12}+p_{21}\right\}
\end{aligned}
$$

We don't have to deconstruct the distribution bounds $L_{i}, U_{i}$ into a thicket $T_{i}$ because the distribution bounds and not the thickets themselves are needed in the next step. 

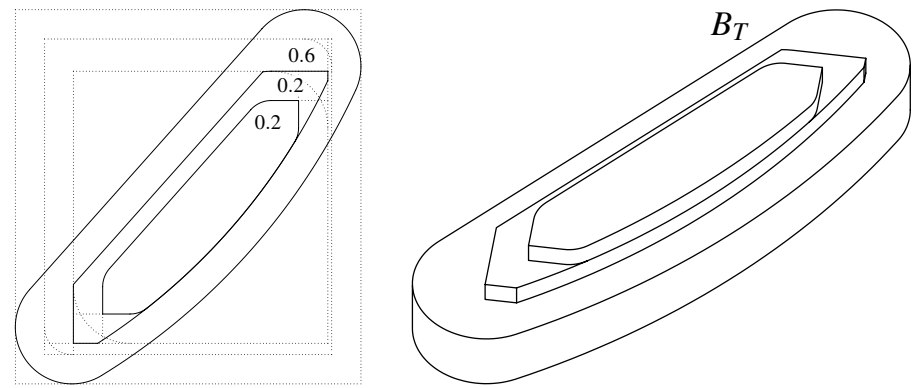

Figure 9. Resulting thicket

\subsection{NESTED THICKET CONVERSION AND INTERSECTION}

We convert the distribution bounds $U_{i}$ and $L_{i}$ into nested thickets and intersect them. In the present example, nested thicket conversion turns out to be information preserving for all four thickets. The resulting thickets consist of two elements with probability mass $0.6,0.2$ and 0.2 , respectively. The result of the intersection according to Theorem 1 is depicted in Figure 9 as set of nested thickets and as nested stack $B_{T}$.

\section{Conclusion}

We extended the univariate DEnv algorithm for bounding the result of arithmetic operations on thickets to the multivariate case. Most concepts turned out to be straightforward generalizations. The multivariate DEnv algorithm is, however, information losing. A further obstacle to the use of DEnv in geometric constructions is its dependence on the coordinate frame.

In order to overcome these drawbacks, we introduced the concept of nested thickets and an intersection algorithm for them, which allows to minimize the effects mentioned above.

Incorporating dependence information as in typical geometric constructions is a topic of future research.

\section{Acknowledgments}

This research was supported by the Austrian Science Foundation (FWF) under grant No. P15911 during the first author's stay at Vienna University of Technology.

\section{References}

Berleant, D. and C. Goodman-Strauss: 1998, 'Bounding the results of arithmetic operations on random variables of unknown dependency using intervals'. Reliab. Comput. 4(2), 147-165. 
Berleant, D., L. Xie, and J. Zhang: 2003, 'Stattool: A tool for Distribution Envelope Determination (DEnv), an interval-based algorithm for arithmetic operations on random variables'. Reliab. Comput. 9(2), 91-108.

Berleant, D. and J. Zhang: 2004a, 'Representation and problem solving with Distribution Envelope Determination'. Reliability Engineering and System Safety 85(1-3), 153-168.

Berleant, D. and J. Zhang: 2004b, 'Using Pearson correlation to improve envelopes around the distributions of functions'. Reliab. Comput. 10(2), 139-161.

$\mathrm{Hu}$, S.-M. and J. Wallner: 2004, 'Error Propagation through Geometric Transformations'. J. Geom. Graphics 8(2), 171-183.

Karloff, H.: 1991, Linear Programming. Boston: Birkhäuser.

Pottmann, H., B. Odehnal, M. Peternell, J. Wallner, and R. Ait Haddou: 2000, 'On optimal tolerancing in Computer-Aided Design'. In: R. Martin and W. Wang (eds.): Geometric Modeling and Processing 2000. Los Alamitos, Calif.: IEEE Computer Society, pp. 347363.

Regan, H. A., S. Ferson, and D. Berleant: 2004, 'Equivalence of methods for uncertainty propagation of real-valued random variables'. Internat. J. Approx. Reason. 36, 1-30.

Requicha, A. A. G.: 1983, 'Towards a theory of geometric tolerancing'. Int. J. of Robotics Research 2, 45-60.

Walley, P.: 1996, 'Measures of uncertainty in expert systems'. Artificial Intelligence 83, $1-58$.

Wallner, J., R. Krasauskas, and H. Pottmann: 2000, 'Error propagation in geometric constructions'. Computer-Aided Design 32, 631-641.

Williamson, R. C. and T. Downs: 1990, 'Probabilistic arithmetic. I. Numerical methods for calculating convolutions and dependency bounds'. Internat. J. Approx. Reason. 4(2), 89-158.

Address for Offprints: Hans-Peter Schröcker, Institut für Technische Mathematik, Geometrie und Bauinformatik, Universität Innsbruck, Technikerstraße 13, A-6020 Innsbruck, Austria 